\title{
Estimation of the organic film thickness on stainless steel using image intensity
}

\author{
Maethinee Songthai ${ }^{1}$, Taweepol Suesut ${ }^{2, *}$, and Navaphattra Nunak $^{1}$ \\ ${ }^{1}$ Food Engineering Department, Faculty of Engineering, King Mongkut's Institute of Technology Ladkrabang, Bangkok, Thailand \\ ${ }^{2}$ Instrumentation and Control Engineering Department, Faculty of Engineering, King Mongkut's Institute of Technology Ladkrabang, \\ Bangkok, Thailand
}

\begin{abstract}
This research proposes an estimation of thickness of organic film coating on a Stainless Steel surface (SS) and a clear microscope slide using a visible optical method with the image intensity level analysis. Sweetened Condensed Milk (SCM) was used as a representative of the organic film. The uncertainty of inverse calibration equations was evaluated to determine the reliability of this technique. The results showed that the image intensity increased with the increasing of the film thickness in a linear relationship with a high coefficient of determination $\left(R^{2}>0.97\right)$. Uncertainty of both inverse calibration equations was low and acceptable. It could be concluded that this technique could be used to estimate the thickness of the organic translucent film coating directly on a SS or a clear slide with the limitation of approximately $1.0 \mathrm{~mm}$ thickness using the reported linear inverse calibration equations.
\end{abstract}

\section{Introduction}

Cleaning of the food contact surface of processing equipment is the most important process in the food industry since it preserves the high quality of food products $[1,2,3]$. The cleaning method should be effective to ensure that all the food residues, dirt, lubricants and foulant on the food contact surface are eliminated [4]. The parameters of cleaning depend on soil types which can be classified into three types, i.e. viscoelastic film such as yogurt and toothpaste (type 1), microbial film (type 2) and solid/cohesive film such as pasteurized milk, sweetened condensed milk (type 3). [2,3] The most difficult soil type for cleaning is the group of cohesive film as it requires the contact time of water for removing the solid layers from the surface with the cohesion and adhesion forces and the chemical action for cleaning. The force that required to break soil from the internal surface of open equipment such as a large tank is a wall shear stress $[5,6]$. Each type of residues needs the different wall shear stress for soil removal causing to the different amount of water required in each cleaning step [5]. An understanding on soil behavior, especially soil film deposited on and removal from the surface during the process is a necessity of cleaning. There are several techniques to measure the film thickness; however, the optical method is the preferable technique which allows a real-time measurement of the organic film thickness [7]. In case of an optical UV-Vis method requires to maintain a constant temperature and $\mathrm{pH}$ of the liquid or cleaning detergent throughout the process [6], while the visible method can detect the fouling removal without any control of temperature and $\mathrm{pH}$ of chemicals [8].
To obtain the amount of soil residues on the surface can be evaluated from the thickness of soil film. Therefore, the thickness measurement should be reliable. As the calibration equation is a part of measurement, it should be taken into account. Several researchers have reported that the mean squared error of an inverse calibration equation is usually smaller than that of a classical calibration equation $[9,10,11,12]$. Besides, the inverse calibration equation is suitable for the small group of samples [13].

Therefore, the objectives of this research are to propose an optical method in the visible region for estimating the thickness of organic soil film on a Stainless Steel surface (SS) using the image intensity level and to evaluate the uncertainty of inverse calibration equation. The Sweetened Condensed Milk (SCM), a protein-sugar based food product, was chosen as the representative of the organic solid/cohesive film.

\section{Materials and methods}

\subsection{Organic soil film preparation and experimental set up}

SCM (Mali Co., Ltd, tube package) was coated as an organic film on a SS 316 having the average roughness value $\left(R_{a}\right)$ of $0.4 \mu \mathrm{m}$ and a clear microscope slide (Sail brand, $1.2 \mathrm{~mm}$ thickness) using the experimental unit (Fig. 1a). The film thickness was adjusted from 0.1 to 10 $\mathrm{mm}$ by adjusting the height of a wiper. The mass of SCM was weighted after coating on the surface for calculating the thickness of SCM film from its density.

\footnotetext{
Corresponding author: taweepol.su@kmitl.ac.th
} 
The experimental unit consisted of a CCD camera (Basler model acA2500-14gc, color 2590 x 1942 pixel) connected with the Gigabit Ethernet (Gig E), a tripod, two light sources (fluorescent lamp, LAMP-TAN, 50-60 Hz, $8 \mathrm{~W}$ ), a control chamber with a black surface, and a computer set as shown in Fig $1 b$.

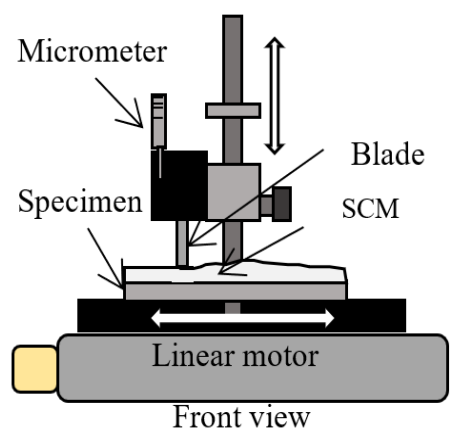

(a)

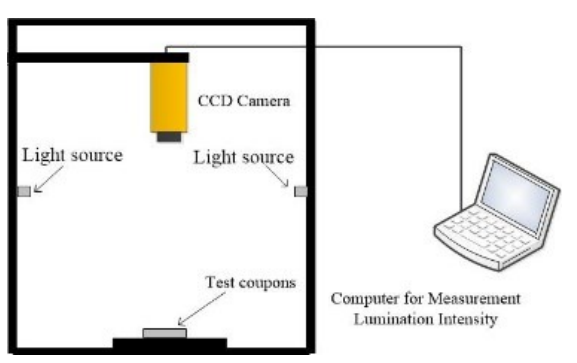

(b)

Fig. 1. Experimental unit

The inverse calibration equations were plotted between the intensity value obtained from an image analysis and the thickness of SCM film which directly coated on a SS and that coated on a clear slide putting on the SS. The examples of test specimens are presented in Fig. 2.

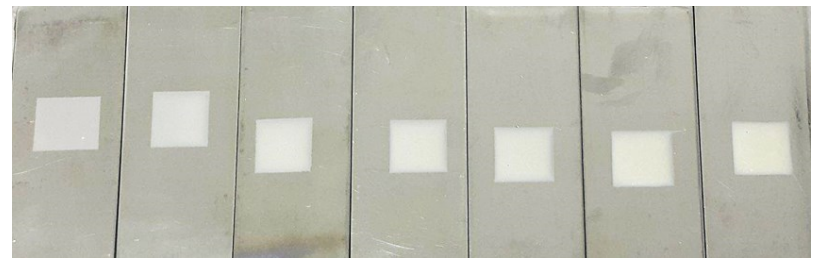

Fig. 2. The SCM film on SS with 0.2 to $0.8 \mathrm{~mm}$ of thickness

\subsection{Determination of an intensity level of the SCM film}

Intensity level of SCM film on the surface was determined by a computer vision system for real-time computing using the Vision Builder Software AI 2014 (National Instrument) by the following steps (Fig. 3):

Step 1: Images were acquired from the object via Gig E and CCD camera in real-time.

Step 2: Region of Interest (ROI) based processing was applied for segmentation of the SCM film area

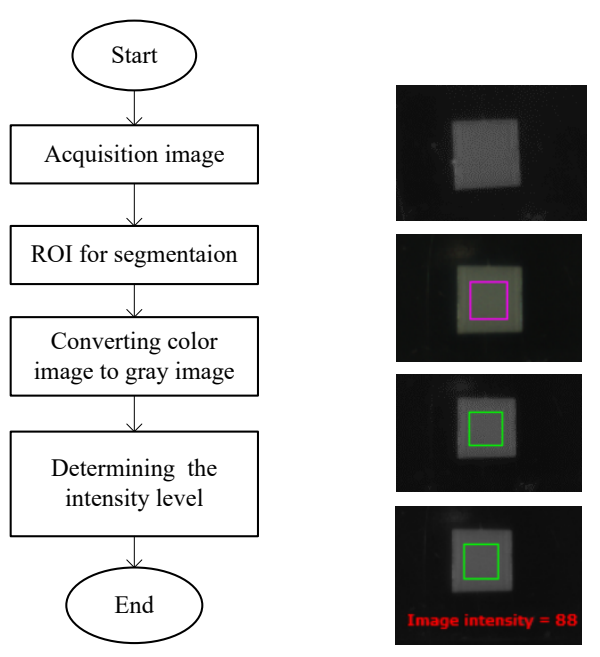

Fig. 3. Determination of image intensity level

Step 3: The color image was converted to grayscale by following steps; comparing the histogram of SCM film with different color model, finding the suitable color model, and extracting color image to gray image. It was found that the lightness from HSL model could identify the intensity level related to the film thickness better than RGB and HSV model.

Step 4: Intensity level was determined by using the measurement intensity, a function in Vision Builder Software.

\subsection{The inverse calibration equation and uncertainty analysis}

The estimation of SCM film thickness from an inverse calibration equation, presenting the relationship between reading values (image intensity) and thickness of SCM film, by the following steps: fitting the inversed calibration curve from the relationship between the image intensity level and the SCM film thickness, predicting the SCM film thickness, and calculating the uncertainty, respectively. The details of each step are described below:

To create an inverse calibration equation, $x$ was fixed as a dependent variable (intensity level value) and $y$ was a standard function or independent variable (film thickness). The calibration equation is

$$
x=g(y)
$$

and the linear calibration equation is

$$
x=b_{0}+b_{1} y_{i}
$$

where $b_{0}$ is an intercept and $b_{1}$ is a slope.

The residual standard deviation $(s(r))$ is calculated by

$$
s(r)=\sqrt{\frac{\sum_{i=1}^{n}\left(y_{i}-\widehat{y_{l}}\right)^{2}}{n-1}}
$$

where $y_{i}$ is the observed value, $\widehat{y}_{l}$ is the predicted value, and $\mathrm{n}$ is the number of calibration points. The uncertainty 
of predicted value from the inverse calibration $(u(x))$ is a type A uncertainty which calculates from the standard deviation of calibration equation $\left(s\left(x_{c}\right)\right)$ in equation (4).

$$
u(x)=s\left(x_{c}\right)=s \sqrt{\frac{1}{p}+\frac{1}{n}+\frac{\left(y_{p r e d}-\bar{y}\right)^{2}}{\sum\left(y_{i}^{2}\right)-\left(\sum y_{i}\right)^{2} / n}}
$$

where $y_{\text {pred }}$ is a predicted value from equation (3), $\bar{y}$ is an average value of $\mathrm{y}, s$ is the standard deviation of calibration equation, $p$ is the numbers of measurement for prediction, $n$ is the number of measurement for calibration equation. The uncertainty of prediction values can be computed by equation (5).

$$
u\left(y_{\text {pred }}\right)=\frac{s\left(x_{c}\right)}{b_{1}}
$$

\section{Results and discussion}

Fig. 4 shows the relationship of intensity image of SCM film coated on a SS and a microscope slide at different thickness values. The image intensity increased with the increasing of the film thickness in a linear relationship. This could be explained by the principle of light which started from two lamps, light sources, generated the incident light on the film surface, and reflected to the camera. The light intensity which reflected from the SCM film to the camera depended on the thickness of film layer. In case of the thin film layer, it performed like a translucent surface which the absorption was much higher than the reflection and caused to the low image intensity level, and vice versa. However, the increasing of image intensity level was limited approximately at the thickness of $1 \mathrm{~mm}$. It seems like at this thickness all incident light was fully reflected to a camera. Although the thickness increased, there was no difference of intensity values.

Linear equations were plotted as presented in equation (6) and (7) for SCM coated on a slide and that coated on a SS, respectively with a high coefficient of determination $\left(R^{2}\right)$. They are called the inverse calibration equations. Slope of both calibration equations are in equal, 0.062 .

$y=0.062 x-5.0377, R^{2}=0.98$

$y=0.062 x-3.8913, R^{2}=0.97$

where $y$ is the SCM film thickness and $x$ is an image intensity level. Besides, it was found that all image intensity analyzed from image of SCM coated on SS was lower than that from the one coated on a clear microscope slide for all measured thickness values since light could incident at the side of a clear microscope slide.

Fig. 5 (a) and (b) shows the residual plots of inverse calibration equations. It could be seen that the residuals of both equations randomly scattered around zero (calibration line). There was no relation between the spread of the residual and an average image intensity. This indicated that the calibration curve could be recognized as a linear model [10].

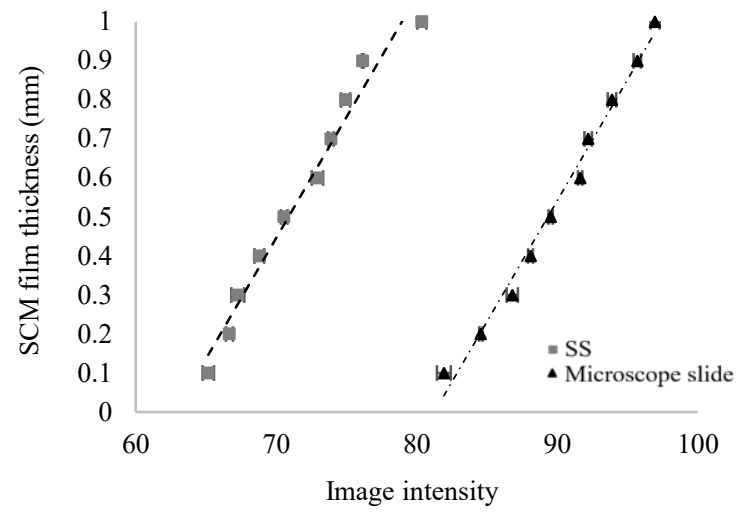

Fig. 4. Appling the inverse calibration to estimate the thickness of SCM film on microscope slides and SS by image intensity level

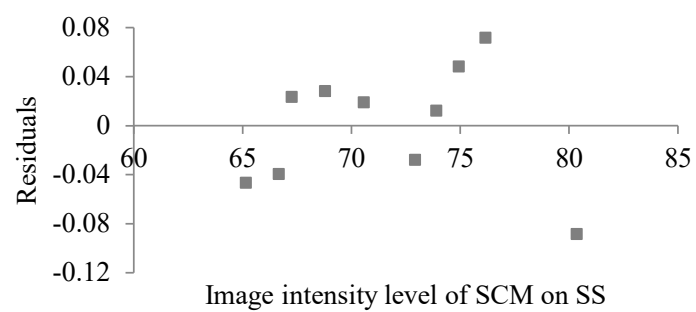

(a)

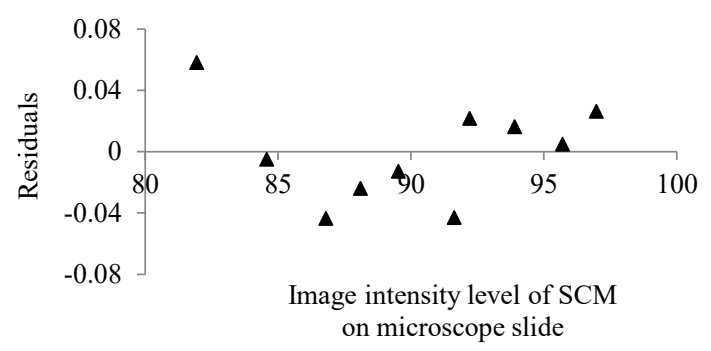

(b)

Fig. 5. Residual plots of inverse calibration equations

The thickness values of SCM film (0.1-1.0 mm) coated on a SS and a clear microscope slide were predicted using the inverse calibration equations as listed in table 1 and also their uncertainty values were evaluated as presented in Fig. 6. It could be observed that the thin layer of SCM coated on the surface $(<0.2 \mathrm{~mm})$ had a high percentage of error. This might be affected from the human error in the procedure of SCM film preparation as the rheological characteristic of SCM is difficult to control in a constant thickness throughout the whole testing surface area. However, when considering uncertainty values, it was found that all evaluated values were acceptable as they were very low and less than the fixed resolutions $(0.1 \mathrm{~mm})$ as it was much enough to investigating and monitoring of the cleaning process. 
Table 1. The prediction values, Standard Deviation (SD) and \% Error of SCM film coated on a SS and a clear microscope slide at different thickness values

\begin{tabular}{|c|c|c|c|c|c|c|}
\hline \multirow{2}{*}{$\begin{array}{c}\text { Measured } \\
\text { thickness } \\
(\mathrm{mm})\end{array}$} & \multicolumn{3}{|c|}{ SS } & \multicolumn{3}{|c|}{ Microscope slide } \\
\hline & $\begin{array}{c}\text { Predicted } \\
\text { thickness } \\
(\mathrm{mm})\end{array}$ & SD & $\%$ Error & $\begin{array}{c}\text { Predicted } \\
\text { thickness } \\
(\mathrm{mm})\end{array}$ & SD & $\%$ Error \\
\hline 0.1 & 0.11 & 0.03 & 9.77 & 0.10 & 0.03 & 1.27 \\
\hline 0.2 & 0.23 & 0.02 & 13.78 & 0.23 & 0.01 & 13.67 \\
\hline 0.3 & 0.28 & 0.03 & 6.92 & 0.27 & 0.01 & 8.44 \\
\hline 0.4 & 0.37 & 0.02 & 6.43 & 0.41 & 0.03 & 1.78 \\
\hline 0.5 & 0.48 & 0.03 & 4.89 & 0.54 & 0.01 & 5.71 \\
\hline 0.6 & 0.59 & 0.02 & 1.79 & 0.62 & 0.02 & 3.33 \\
\hline 0.7 & 0.69 & 0.01 & 1.36 & 0.70 & 0.02 & 0.09 \\
\hline 0.8 & 0.76 & 0.01 & 5.16 & 0.78 & 0.02 & 3.13 \\
\hline 0.9 & 0.96 & 0.00 & 3.11 & 0.86 & 0.02 & 4.24 \\
\hline 1 & 1.09 & 0.02 & 4.14 & 1.00 & 0.02 & 0.39 \\
\hline
\end{tabular}

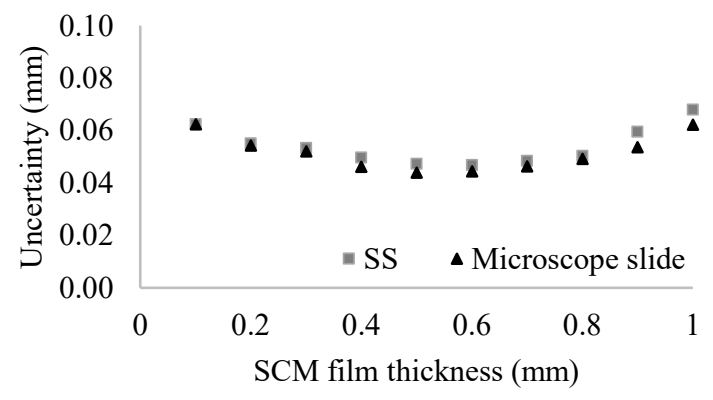

Fig. 6. The uncertainty of SCM film coated on a SS and a clear microscope slide at various thickness values

For these reasons, it could imply that the visible region could be applied to estimate the thickness of an organic film within the limitation of approximately $1.0 \mathrm{~mm}$ using the linear inverse calibration equation. The SCM film could be prepared by coating directly on a SS or coated on a clear slide putting on a SS.

\section{Conclusions}

An optical method in the visible region, an inexpensive and simple technique, proposed in this research with the inverse calibration equations obtained from performing the experiments by coating SCM film directly on a SS and on a clear slide was capable to estimate the thickness of organic film. Nevertheless, this technique was limited only for the translucent film which the absorption and reflection change according to changes of the thickness and the maximum thickness was approximately $1.0 \mathrm{~mm}$. In addition, it was the technique having a great potential for improving to the real-time estimation. However, the further research is needed to improve the film preparation and investigate the other sources of uncertainty to calculate the combined uncertainty of this technique.

\section{Reference}

1. A.Y. Tamime, Cleaning-in-Place: Dairy, Food and Beverage Operations Third Edition. Blackwell Publishing, (2008)

2. K. R. Goode, K. Asteriadou, P. T. Robbins, P. J. Fryer, "Fouling and cleaning studies in the food and beverage industry classified by cleaning type," Institute of food technologists, 12, 121-143, (2013)

3. P.J. Fryer, and K. Asteriadou, "A prototype cleaning map: A classification of industrial cleaning process." Trends in food Science \& Technology, 20, 255 - 262, (2009)

4. EHEDG. (2004). "Document No. 8: Hygienic Equipment Design Criteria ( $\left.2^{\text {nd }} E d.\right)$ ".

5. E. Fuchs, A. Boye, H. Stoye, M. Mauermann and J.-P. Majschak, "Influence of the film flow characteristic on the cleaning behavior." International Conference on Heat Exchanger Fouling and Cleaning. 411-420E. (2013)

6. E. Fuchs, R. Boye, J.-Murcek, P. Majschak, “An experimental comparison of film flow parameters and cleaning behavior of falling liquid film for different tilt angles," Food and bioprocessing, 93, 318-326, (2015)

7. M. Mauermann, U. Eschenhagen, T. Wey-rauch, H. Köhler, Th. Bley, J. P. Majschak. "Monitoring the progress of cleaning using optical detection methods" Fouling and Cleaning in Food Processing, (2010)

8. N. I. Khalid, N. Nordin, N. Aziz, A. A. Ab. Nuraini, F.S. Taip, and A. Ahmedov, "Removal kinetic of pink guava puree fouling deposit from a stainless surface during alkaline cycle." Proceedings of International on Heat Exchanger fouling and cleaning, 396-404, (2015)

9. B. Klongratog, T. Suesut, and N. Nunak. "The uncertainty in sugar solution concentration measurement based on density approach" Advanced Materials Research, 811, 358-364, (2013)

10. L. Tzehung, and C. Chaichung. "Uncertainty evaluation of humidity sensors calibrated by saturated salt solutions", Measurement, 40, 591599, (2007)

11. R. G. Krutchkoff, "Classical and inverse regression methods of calibration", Technometrics, 9 (3), 425-439, (1967)

12. V. Centner, D.L. Massart, S. de Jong, "Inverse calibration predicts better than classical calibration", Fresenius' Journal of Analytical Chemistry., 361, 2-9, (1998)

13. J. Tellinghuisen, "Inverse vs. classical calibration for small data sets", Fresenius J Anal Chem. 368 :585-588, (2000) 